\title{
NOVEL METHOD FOR REDUCTION OF WAVELET COEFFICIENTS NUMBER AND ITS APPLICATIONS IN IMAGES COMPRESSION
}

\author{
Bachir Dehda ${ }^{a}$ and Khaled Melkemi ${ }^{b}$ \\ aDepartment of Mathematics, University of Mohamed Khider, Biskra,7000 Algeria \\ aDepartment of Mathematics, University of Echahid Hamma Lakhdar, Eloued, \\ 39000 Algeria \\ bDepartment of Mathematics, University of Elhadj Lakhdar, Batna 5000, Algeria
}

\begin{abstract}
In this paper, we present a new method for images compression, which is based on a reduction of the wavelet coefficients number independently of the singularity contained in the images. This method, called the hybridization of detail space (HDS), compared to discrete wavelet transform (DWT), it has many advantages. The (HDS) method considers first an $r$-regular multiresolution analysis $\left(V_{j}\right)_{j \in \mathbb{Z}}$ of $L^{2}(\mathbb{R})$ and replaces a great number of its wavelet basis elements by new vectors using the orthogonal matrices and prime numbers. So, it can make a great number of wavelet coefficients be equal to zero independently of the image singularity. For illustrating the effectiveness of the (HDS) method compared to (DWT), we have implemented the algorithm on a gray-scale image that has considerable singularities in MATLAB, and the decomposition has been done at level $j=1$ using Haar wavelet. So, the experimental results with this new method have given the better performance for the quality and the size of the compressed image.
\end{abstract}

\footnotetext{
${ }^{*}$ Corresponding author.

E-mail address: bachirdehda@gmail.com (Bachir Dehda).

Copyright (C) 2016 Scientific Advances Publishers

2010 Mathematics Subject Classification: 94A08, 68U10, 62H35, 65T60.

Submitted by Hind Rustum Mohammed Shaaban.

Received July 21, 2016; Revised August 17, 2016
} 
Keywords: wavelets, multiresolution analysis, hybridization of detail space, image compression and denoising.

\section{Introduction}

Image compression techniques are the important research topics today, the transformation compression methods are classified with irreversible techniques and they are the most widely used of recent years. These methods decompose the image into an orthonormal basis, such as we can make a great number of its coefficients be equal to zero, without significantly impairing the visual aspect of the original image. Factually, the wavelet theory has given high contributions in the transformation compression methods, that has evolved in the signal processing domain since 1985 by birthing of the multiresolution analysis (MRA) provided by Mallat [7] and Meyer [10], which is considered as an approximative approach for construct an orthonormal basis of $L^{2}\left(\mathbb{R}^{n}\right)$ as $\left\{2^{\frac{n j}{2}} \psi\left(2^{j} x-k\right)\right\}_{k \in \mathbb{Z}^{n}, j \in \mathbb{Z}}$. These bases can be chosen for to show theirs abilities in the images compression, such as to make a more great number of wavelet coefficients be equal to zero with respecting the significative visual aspect of the original image. So, there are only few numbers of those coefficients for representing the necessary informations of the image as well as Daubechies wavelets [5] and Coifman wavelets [3].

This ability is referred to nice mathematical properties of the wavelet as: compactly supported, vanished moments and smoothness, but if that image has a large singularity, we will be obliged to increase the number of non-zero wavelet coefficients in the image approximation. In fact, this situation has led to an emergence of the non-stationary wavelet [2]; which is based on changing the wavelet filter $H$ at every level $j$, and wavelet packets [9] that approximates the detail space $W_{j}$ by decomposing it under the form $W_{j}=\underset{\varrho}{\bigoplus} W_{j, \varrho}$. 
However, all last kinds of wavelets are related to previous mathematical properties, then the decreasing of the number of non-zero wavelet coefficients depends on the singularity of the image. So that, in the case of the singularity is large enough, we will have a great number of grand wavelet coefficients needed to representing the approximated image. Hence, those wavelets are not sufficient to solving the images compression problem. For that, our goal depends on to answer the following question: are there wavelet bases can reduce their wavelet coefficients number independently of the singularity contained in the image? In fact, the hybridization of detail space method (HDS) is a technique that works to replace a great number of $\left\{\psi_{j, k}\right\}$ by new elements using only the orthogonal matrices and prime numbers. So, it can make a great number of wavelet coefficients be equal to zero independently of the image singularity.

Our work is structured as follows: Section 2 presents the (HDS) method by introducing some necessary definitions and basic theorems, which are associated with advantages of this new method compared to discrete wavelet transform. Section 3 describes the decomposition of signal in the second-dimensional case and the corresponding decomposition scheme at level $j$. Section 4 illustrates the principal steps and the flowchart of the compression algorithm corresponding to the (HDS) method. Section 5 gives the compression results of a gray-scale image and discussing them. Finally, we close this work by a conclusion and main references.

\section{Reduction of Wavelet Coefficients Number Method}

The hybridization of detail space method (HDS) is a novel method for reducing of the wavelet coefficients number independently of the singularity existed in the signal. This technique is based on a decomposition of the detail space $W_{j}$ using the orthogonal matrices and prime numbers for getting a great number of null wavelet coefficients. So that, we will obtain a nice compressed signal quality. In the following subsections, we present theoretical aspects of the (HDS) method. 


\subsection{Theoretical concept of the hybrid detail space}

In this subsection, we start by giving the theoretical concept of the hybrid detail space.

Definition 1. Let $\left(V_{j}\right)_{j \in \mathbb{Z}}$ be an $r$-regular multiresolution analysis of $L^{2}(\mathbb{R})$ characterised with its wavelet function $\psi$. The hybrid detail space of $W_{j}$ is a subspace of $W_{j}$ noted $W_{j}^{H}$ and defined as follows:

$$
\left.W_{j}^{H}=\overline{\operatorname{span}\left\{\Psi_{j, p}^{(i)} ; 1 \leq i \leq N_{j, p}, p\right. \text { is a prime number }}\right\}
$$

such as:

$$
\left(\begin{array}{c}
\Psi_{j, p}^{(1)} \\
\Psi_{j, p}^{(2)} \\
. \\
\Psi_{j, p}^{(k)} \\
\ldots \\
\Psi_{j, p}^{\left(N_{j, p}\right)}
\end{array}\right)=A_{j, p}\left(\begin{array}{c}
\psi_{j, p} \\
\psi_{j, 2 p} \\
\cdots \\
\psi_{j, 2^{k-1} p} \\
\cdots \\
\psi_{j, 2^{N_{j, p}-1} p}
\end{array}\right),
$$

where $A_{j, p}$ is an orthogonal matrix of $\mathcal{M}_{N_{j, p}}(\mathbb{R})$ and $N_{j, p}$ is an integer number.

Remark 2. From the formula (1), we can write the elements $\left\{\Psi_{j, p}^{(i)}\right\}_{i=1}^{N_{j, p}}$ as:

$$
\Psi_{j, p}^{(i)}=\sum_{k=1}^{N_{j, p}} r_{(j, p)}^{(i)}(k) \psi_{j, 2^{k-1} p} ; \quad 1 \leq i \leq N_{j, p}
$$

Theorem 3. The family $\left\{\Psi_{j, p}^{(i)} ; 1 \leq i \leq N_{j, p}, p\right.$ is a prime number $\}$ defined in (1) is an orthonormal basis of $W_{j}^{H}$. 
Proof. It is enough to prove an orthonormality between the vectors $\left\{\Psi_{j, p}^{(i)}\right\}$. Let $p$ and $q$ be prime numbers and for $i$; $\varrho$ such as $1 \leq i \leq N_{j, p}$, $1 \leq \varrho \leq N_{j, q}$, we have

$$
\begin{aligned}
\left\langle\Psi_{j, p}^{(i)}, \Psi_{j, q}^{(\varrho)}\right\rangle & =\sum_{k, l=1}^{N_{j, p}, N_{j, q}} r_{(j, p)}^{(i)}(k) r_{(j, q)}^{(\varrho)}(l)\left\langle\psi_{j, 2^{k-1} p}, \psi_{j, 2^{l-1} q}\right\rangle \\
& =\sum_{k, l=1}^{N_{j, p}, N_{j, q}} r_{(j, p)}^{(i)}(k) r_{(j, q)}^{(\varrho)}(l) \delta_{2^{k-1} p, 2^{l-1} q},
\end{aligned}
$$

we want to prove that $2^{k-1} p=2^{l-1} q$ if and only if $k=l$ and $p=q$, for all $k, l, p, q$. For that, we assume that $2^{k-1} p=2^{l-1} q$ and $k \neq l$, in this case, the prime number $p$ or $q$ will divide the another prime number. Consequently, this is a contradiction with the fact that $p$ and $q$ are prime numbers. Hence, we get

$$
\begin{aligned}
\left\langle\Psi_{j, p}^{(i)}, \Psi_{j, q}^{(\varrho)}\right\rangle & =\sum_{k, l=1}^{N_{j, p}, N_{j, q}} r_{(j, p)}^{(i)}(k) r_{(j, q)}^{(\varrho)}(l) \delta_{k, l} \delta_{p, q} \\
& =\sum_{k, l=1}^{N_{j, p}, N_{j, q}} r_{(j, p)}^{(i)}(k) r_{(j, q)}^{(\varrho)}(l) \delta_{k, l} \delta_{p, q} \\
& =\delta_{p, q} \delta_{i, \varrho} .
\end{aligned}
$$

Finally, the family $\left\{\Psi_{j, p}^{(i)} ; 1 \leq i \leq N_{j, p}, p\right.$ is a prime number $\}$ is an orthonormal basis of $W_{j}^{H}$.

Theorem 4. Let $W_{j}^{H}$ be a hybrid detail space of $W_{j}$, then

$$
W_{j}=W_{j}^{H} \oplus W_{j}^{H \perp},
$$


where

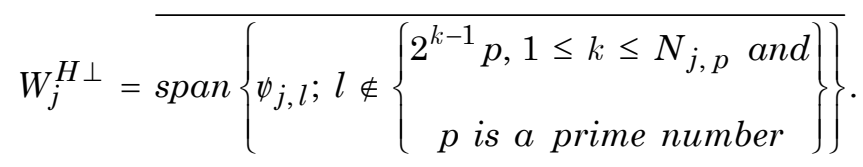

So, the family

$$
\begin{gathered}
\left\{\Psi_{j, p}^{(i)} ; 1 \leq i \leq N_{j, p}, p \text { is a prime number }\right\} \\
\cup\left\{\psi_{j, l} ; l \notin\left\{\begin{array}{c}
2^{k-1} p, 1 \leq k \leq N_{j, p} \text { and } \\
p \text { is a prime number }
\end{array}\right\}\right\}
\end{gathered}
$$

is an orthonormal basis of $W_{j}$.

Proof. Since $W_{j}^{H}$ is a closed subspace of the Hilbert space $W_{j}$, then according to the orthogonal projection theorem, we obtain the relation (3). In another side, the formula (1) provides

$$
\left(\begin{array}{c}
\psi_{j, p} \\
\psi_{j, 2 p} \\
. \\
\psi_{j, 2^{k-1} p} \\
. \\
\psi_{j, 2_{j, p}{ }^{N_{j, p}}}
\end{array}\right)=A_{j, p}^{-1}\left(\begin{array}{c}
\Psi_{j, p}^{(1)} \\
\Psi_{j, p}^{(2)} \\
\ldots \\
\Psi_{j, p}^{(k)} \\
\cdots \\
\Psi_{j, p}^{\left(N_{j, p}\right)}
\end{array}\right),
$$

this implies that every element of the family

$$
\left\{\psi_{j, 2^{k-1} p} ; 1 \leq k \leq N_{j, p}, p \text { is a prime number }\right\} \text { can be written as }
$$
linear combinations of vectors $\left\{\Psi_{j, p}^{(i)} ; 1 \leq i \leq N_{j, p}, p\right.$ is a prime number $\}$. Furthermore, for $l \notin\left\{\begin{array}{c}2^{k-1} p, 1 \leq k \leq N_{j, p} \text { and } \\ p \text { is a prime number }\end{array}\right\}$ and $1 \leq i \leq N_{j, q}, q$ is a prime number, we have 


$$
\left\langle\Psi_{j, q}^{(i)}, \psi_{j, l}\right\rangle=0
$$

So, the family

$$
\begin{aligned}
& \left\{\Psi_{j, p}^{(i)} ; 1 \leq i \leq N_{j, p}, p \text { is a prime number }\right\} \\
& \cup\left\{\psi_{j, l} ; l \notin\left\{\begin{array}{c}
2^{k-1} p, 1 \leq k \leq N_{j, p} \text { and } \\
p \text { is a prime number }
\end{array}\right\}\right\}
\end{aligned}
$$

is an orthonormal basis of $W_{j}$.

Through this theorem, we observe that the orthogonal projection of any signal of $L^{2}(\mathbb{R})$ on $W_{j}$ can be expressed as a sum of two orthogonal projections on $W_{j}^{H}$ and $W_{j}^{H \perp}$.

Corollary 5. Let $\left(W_{j}^{H}\right)_{j \geq 0}$ be a sequence of hybrid detail spaces of $L^{2}(\mathbb{R})$, then we can decompose $L^{2}(\mathbb{R})$ as:

$$
L^{2}(\mathbb{R})=V_{0} \oplus\left(\bigoplus_{j \geq 0}\left(W_{j}^{H} \oplus W_{j}^{H \perp}\right)\right) .
$$

So, for all function $f \in L^{2}(\mathbb{R})$, we have

$$
f=P_{V_{0}} f+\sum_{\substack{j \geq 0 \\ \text { pis a prime number } \\ l \notin\left\{2^{k-1} p, 1 \leq k \leq N_{j, p}\right\}}} d_{j, l} \psi_{j, l}+\sum_{\substack{j \geq 0 \\ \text { pis a prime number } \\ 1 \leq i \leq N_{j, p}}} D_{j, p}^{(i)} \Psi_{j, p}^{(i)},
$$

where $P_{V_{0}} f$ is an orthogonal projection of $f$ on $V_{0}, d_{j, k}=\int_{\mathbb{R}} f(x) \psi_{j, k}(x) d x$ and $D_{j, p}^{(i)}=\int_{\mathbb{R}} f(x) \Psi_{j, p}^{(i)}(x) d x$.

Proof. If we apply the precedent theorem and taking into account that $L^{2}(\mathbb{R})=V_{0} \oplus\left(\bigoplus_{j \geq 0} W_{j}\right)$, this corollary will be immediate. 
Remark 6. We can define $W_{j}^{H}$ for a finite number of prime numbers as: $W_{j}^{H}=\overline{\operatorname{span}\left\{\Psi_{j, p}^{(i)} ; 1 \leq i \leq N_{j, p}, p \text { is a prime number such as } N_{1} \leq p \leq N_{2}\right\}}$.

The following theorem characterises the hybrid detail space:

Theorem 7. Let $W_{j}^{H}$ be a hybrid detail space of $W_{j}$ and $\alpha \in \mathbb{R}^{*}$, then for every prime number $p$, it exists an orthogonal matrix $A_{j, p, \alpha}$ of $\mathcal{M}_{N_{j, p}}(\mathbb{R})$ verifies:

$$
A_{j, p, \alpha}\left(\begin{array}{c}
d_{j, p} \\
d_{j, 2 p} \\
\ldots \\
\ldots \\
d_{j, 2^{N_{j, p}-1} p}
\end{array}\right)=\left(\begin{array}{c}
D_{j, p}^{(1)} \\
D_{j, p}^{(2)} \\
\ldots \\
\ldots \\
D_{j, p}^{\left(N_{j, p}\right)}
\end{array}\right)=\left(\begin{array}{c}
D_{j, p}^{(1)} \\
0 \\
\ldots \\
\ldots \\
0
\end{array}\right) .
$$

For proof of this theorem, we need to introduce the following lemma:

Lemma 8. Let $U=\left(u_{1}, u_{2}, \ldots, u_{N}\right)^{T}$ be a vector of $\mathcal{M}_{N, 1}(\mathbb{R})-\{0\}$ and $\alpha \in \mathbb{R}^{*}$, then it exists an orthogonal matrix $A_{\alpha}$ of $\mathcal{M}_{N}(\mathbb{R})$ verifies:

$$
A_{\alpha} U=V_{\alpha}
$$

where $V_{\alpha}=\left(v_{\alpha, 1}, v_{\alpha, 2}, \ldots, v_{\alpha, N}\right)^{T}$ is a vector of $\mathcal{M}_{N, 1}(\mathbb{R})$ such that $v_{\alpha, 1} \neq 0$ and $v_{\alpha, i}=0$ for every $i=2, \ldots, N$.

Proof of Lemma 8. Let $X=\left(x_{1}, x_{2}, \ldots, x_{N}\right)^{T}$ be a vector of $\mathcal{M}_{N, 1}(\mathbb{R})$ such as: $x_{1}=\alpha$ and $x_{i}=0$ for every $i=2, \ldots, N$. 
For $B=X U^{T} \in \mathcal{M}_{N}(\mathbb{R})$, then it can be written as:

$$
B=\left(\begin{array}{cccc}
\alpha u_{1} & \alpha u_{2} & \ldots & \alpha u_{N} \\
0 & 0 & \ldots & 0 \\
\cdots & \ldots & \ldots & \ldots \\
0 & 0 & \ldots & 0
\end{array}\right) .
$$

Hence, applying the singular values decomposition on $B$, it results that

$$
B=P Q R^{T},
$$

where $P$ and $Q$ are, respectively, an orthogonal and a diagonal matrix of $\mathcal{M}_{N}(\mathbb{R})$ such that the $N-1$ back columns of $R, Y_{2}, \ldots, Y_{N}$ that are obtained in the following way:

$$
B^{T} B Y_{i}=0 ; \quad \forall i=2, \ldots, N,
$$

because $\operatorname{rank}(B)=\operatorname{rank}\left(B^{T} B\right)=\operatorname{rank}\left(B B^{T}\right)=1$. In another side, we have

$$
B^{T} B=\alpha^{2}\left(\begin{array}{ccccc}
u_{1}^{2} & u_{1} u_{2} & \ldots & \ldots & u_{1} u_{N} \\
u_{2} u_{1} & u_{2}^{2} & \ldots & \ldots & u_{2} u_{N} \\
\ldots & \ldots & \ldots & \ldots & \ldots \\
u_{i} u_{1} & \ldots & u_{i}^{2} & \ldots & u_{i} u_{N} \\
u_{N} u_{1} & \ldots & \ldots & \ldots & u_{N}^{2}
\end{array}\right) .
$$

If we put $Y_{i}=\left(\beta_{1}^{i}, \beta_{2}^{i}, \ldots, \beta_{N}^{i}\right)$ for every $i=2, \ldots, N$, we will obtain 


$$
\left\{\begin{array}{cc}
u_{1}^{2} \beta_{1}^{i}+u_{1} u_{2} \beta_{2}^{i}+\ldots+u_{1} u_{N} \beta_{N}^{i} & =0 \\
u_{2} u_{1} \beta_{1}^{i}+u_{2}^{2} \beta_{2}^{i}+\ldots+u_{2} u_{N} \beta_{N}^{i} & =0 \\
\ldots \ldots & \cdots \\
\ldots \ldots & \cdots \\
u_{i} u_{1} \beta_{1}^{i}+u_{i} u_{2} \beta_{2}^{i}+. .+u_{i}^{2} \beta_{i}^{i}+. .+u_{i} u_{N} \beta_{N}^{i} & =0 \\
\ldots \ldots & \cdots \\
u_{N} u_{1} \beta_{1}^{i}+u_{N} u_{2} \beta_{2}^{i}+\ldots+u_{N}^{2} \beta_{N}^{i} & =0 .
\end{array}\right.
$$

After this system, we conclude that

$$
u_{j}\left(\sum_{k=1}^{N} u_{k} \beta_{k}^{i}\right)=0 \text {; for every } j=1, \ldots, N \text { and } i=2, \ldots, N .
$$

Since $U \neq 0$, then it exists $u_{j_{0}} \neq 0$ such that $u_{j_{0}}\left(\sum_{k=1}^{N} u_{k} \beta_{k}^{i}\right)=0$. Hence, $\sum_{k=1}^{N} u_{k} \beta_{k}^{i}=0$. Consequently, if we take $A_{\alpha}=R^{T}$, we will find that $v_{\alpha, i}=0$ for every $i=2, \ldots, N$ and $v_{\alpha, 1} \neq 0$, because $\left\|A_{\alpha} U\right\|=\|U\| \neq 0$.

Proof of Theorem 7. If we take $U=\left(d_{j, p}, d_{j, 2 p}, \ldots, d_{j, 2^{N_{j, p}-1} p}\right)^{T}$, then in case of $U \neq 0$, a proof of the theorem will be immediate from previous lemma. The another case, for $U=0$, all orthogonal matrices verify $A_{j, p, \alpha} U=0$.

From the previous theorem, we can conclude a very basic advantage concerning the hybrid wavelet coefficients, that means that the number of hybrid wavelet coefficients is equal to the number of used prime numbers. 


\subsection{Advantages of (HDS) Method compared to discrete wavelet transform}

In this subsection, we will display very basic advantages of the hybridization of detail space method compared to discrete wavelet transform.

(1) By choosing an appropriate orthogonal matrix for each prime number as in the latest precedent subsection theorem, the hybridization of detail space method can make a great number of hybrid wavelet coefficients be equal to zero in the signal decomposition independently of the singularity existed in this signal, whereas the discrete wavelet transform can not make the wavelet coefficients which are depended on the zone where that signal has large singularities be equal to zero.

(2) The hybridization of detail space method can compute the number of non-zero hybrid wavelet coefficients by choosing the number of prime numbers as in the latest precedent subsection remark, but the discrete wavelet transform has not this property for its wavelet coefficients.

\section{Decomposition of Signal in Second-Dimensional Case}

Before presenting the novel decomposition of a signal in seconddimensional case, we need to the following theorem:

Theorem 9. Let $\left(V_{j}^{1}\right)_{j \in \mathbb{Z}}$ be an r-regular multiresolution analysis of $L^{2}(\mathbb{R})$ characterised with its scaling and wavelet function $\varphi$ and $\psi$, we consider a separable multiresolution analysis of $L^{2}\left(\mathbb{R}^{2}\right)$ associated to $\left(V_{j}^{1}\right)_{j \in \mathbb{Z}}$, then the family

$$
\begin{array}{r}
\left\{\varphi_{j, k}(x) \Psi_{j, p}^{(i)}(y) ; 1 \leq i \leq N_{j, p}, p \text { is a prime number, } k \in \mathbb{Z}\right\} \\
\cup\left\{\varphi_{j, k}(x) \psi_{j, l}(y) ; l \notin\left\{\begin{array}{c}
2^{n-1} p, 1 \leq n \leq N_{j, p} \text { and } \\
p \text { is a prime number }
\end{array}\right\}, k \in \mathbb{Z}\right\}
\end{array}
$$




$$
\begin{aligned}
& \cup\left\{\Psi_{j, p}^{(i)}(x) \varphi_{j, k}(y) ; 1 \leq i \leq N_{j, p}, p \text { is a prime number, } k \in \mathbb{Z}\right\} \\
& \cup\left\{\psi_{j, l}(x) \varphi_{j, k}(y) ; l \notin\left\{\begin{array}{c}
2^{n-1} p, 1 \leq n \leq N_{j, p} \text { and } \\
p \text { is a prime number }
\end{array}\right\}, k \in \mathbb{Z}\right\} \\
& \cup\left\{\psi_{j, k}(x) \Psi_{j, p}^{(i)}(y) ; 1 \leq i \leq N_{j, p}, p \text { is a prime number, } k \in \mathbb{Z}\right\} \\
& \cup\left\{\psi_{j, k}(x) \psi_{j, l}(y) ; l \notin\left\{\begin{array}{c}
2^{n-1} p, 1 \leq n \leq N_{j, p} \text { and } \\
p \text { is a prime number }
\end{array}\right\}, k \in \mathbb{Z}\right\}
\end{aligned}
$$

is an orthonormal basis of $W_{j}$.

Proof. It is well known that of [10], all detail spaces in the seconddimensional case for a separable multiresolution analysis of $L^{2}\left(\mathbb{R}^{2}\right)$ are decomposed as:

$$
W_{j}=\left(V_{j}^{1} \otimes W_{j}^{1}\right) \oplus\left(W_{j}^{1} \otimes V_{j}^{1}\right) \oplus\left(W_{j}^{1} \otimes W_{j}^{1}\right) .
$$

Furthermore, after the following decompositions:

$$
\begin{aligned}
V_{j}^{1} \otimes W_{j}^{1} & =V_{j}^{1} \otimes\left(W_{j}^{1 H} \oplus W_{j}^{1 H \perp}\right) \\
& =\left(V_{j}^{1} \otimes W_{j}^{1 H}\right) \oplus\left(V_{j}^{1} \otimes W_{j}^{1 H \perp}\right) ; \\
W_{j}^{1} \otimes V_{j}^{1} & =\left(W_{j}^{1 H} \oplus\left(W_{j}^{1 H}\right)^{\perp}\right) \otimes V_{j}^{1} \\
& =\left(W_{j}^{1 H} \otimes V_{j}^{1}\right) \oplus\left(W_{j}^{1 H \perp} \otimes V_{j}^{1}\right) ; \\
W_{j}^{1} \otimes W_{j}^{1} & =W_{j}^{1} \otimes\left(W_{j}^{1 H} \oplus W_{j}^{1 H \perp}\right) \\
& =\left(W_{j}^{1} \otimes W_{j}^{1 H}\right) \oplus\left(W_{j}^{1} \otimes W_{j}^{1 H \perp}\right),
\end{aligned}
$$

the theorem is satisfied. 
Corollary 10. The orthogonal projection of a signal $f \in L^{2}\left(\mathbb{R}^{2}\right)$ on $V_{j}$ is expressed by the following decomposition formula:

$$
\begin{aligned}
P_{V_{j}} f= & P_{V_{j-1}} f+P_{V_{j-1}^{1} \otimes W_{j-1}^{1 H} f+P_{V_{j-1}^{1} \otimes W_{j-1}^{1 H \perp}} f+P_{W_{j-1}^{1 H} \otimes V_{j-1}^{1}} f} \\
& +P_{W_{j-1}^{1 H \perp} \otimes V_{j-1}^{1}} f+P_{W_{j-1}^{1} \otimes W_{j-1}^{1 H}} f+P_{W_{j-1}^{1} \otimes W_{j-1}^{1 H \perp}} f .
\end{aligned}
$$

The corresponding decomposition scheme at level $j$ of the hybridization of detail space method is represented below in Figure 1:

\begin{tabular}{|c|c|c|c|}
\hline \multicolumn{2}{|c|}{$V_{j-1}$} & $W_{j-1}^{1 H} \otimes V_{j-1}^{1}$ & $W_{j-1}^{1 H \perp} \otimes V_{j-1}^{1}$ \\
\hline$V_{j-1}^{1} \otimes W_{j-1}^{1 H}$ & $V_{j-1}^{1} \otimes W_{j-1}^{1 H \perp}$ & $W_{j-1}^{1} \otimes W_{j-1}^{1 H}$ & $W_{j-1}^{1} \otimes W_{j-1}^{1 H \perp}$ \\
\hline
\end{tabular}

Figure 1. Decomposition scheme at level $j$ with the (HDS) method.

\section{Compression Algorithm Based on the (HDS) Method}

This section illustrates the algorithm work of the (HDS) method through the following subsections:

\subsection{Characterisation of hybrid wavelet coefficients}

In this subsection, we characterise the hybrid wavelet coefficients of a signal $f \in L^{2}\left(\mathbb{R}^{2}\right)$ by decomposing them into the classic wavelet coefficients.

Let $D I_{j}^{(1)}, D I_{j}^{(2)}, D I_{j}^{(3)}$ be the classic wavelet coefficients of the detail spaces, respectively, $V_{j}^{1} \otimes W_{j}^{1}, W_{j}^{1} \otimes V_{j}^{1}, W_{j}^{1} \otimes W_{j}^{1}$ such as:

$$
\begin{aligned}
& D I_{j,(n, m)}^{(1)}=\iint_{\mathbb{R}^{2}} f(x, y) \varphi_{j, n}(x) \psi_{j, m}(y) d x d y, \\
& D I_{j,(n, m)}^{(2)}=\iint_{\mathbb{R}^{2}} f(x, y) \varphi_{j, n}(x) \varphi_{j, m}(y) d x d y,
\end{aligned}
$$




$$
D I_{j,(n, m)}^{(3)}=\iint_{\mathbb{R}^{2}} f(x, y) \psi_{j, n}(x) \psi_{j, m}(y) d x d y,
$$

then the hybrid wavelet coefficients, $D H_{j}^{(i, 1)}, D H_{j}^{(i, 2)}, D H_{j}^{(i, 3)}$, respectively, of $V_{j}^{1} \otimes W_{j}^{1 H}, W_{j}^{1 H} \otimes V_{j}^{1}, W_{j}^{1} \otimes W_{j}^{1 H}$ can be written as follows:

$$
\begin{aligned}
& D H_{j,(k, p)}^{(i, 1)}=\iint_{\mathbb{R}^{2}} f(x, y) \varphi_{j, k}(x) \Psi_{j, p}^{(i)}(y) d x d y=\sum_{n=1}^{N_{j, p}} r_{j, p}^{(i)}(n) D I_{j,\left(k, 2^{n-1} p\right)}^{(1)}, \\
& D H_{j,(k, p)}^{(i, 2)}=\iint_{\mathbb{R}^{2}} f(x, y) \Psi_{j, p}^{(i)}(x) \varphi_{j, k}(y) d x d y=\sum_{n=1}^{N_{j, p}} s_{j, p}^{(i)}(n) D I_{j,\left(2^{n-1} p, k\right)}^{(2)}, \\
& D H_{j,(k, p)}^{(i, 3)}=\iint_{\mathbb{R}^{2}} f(x, y) \varphi_{j, k}(x) \Psi_{j, p}^{(i)}(y) d x d y=\sum_{n=1}^{N_{j, p}} t_{j, p}^{(i)}(n) D I_{j,\left(k, 2^{n-1} p\right)}^{(3)},
\end{aligned}
$$

where the hybrid detail space $W_{j}^{1 H}$ is changed in each following detail space: $V_{j}^{1} \otimes W_{j}^{1}, W_{j}^{1} \otimes V_{j}^{1}, W_{j}^{1} \otimes W_{j}^{1}$ for the purpose of achieving the reduction of hybrid wavelet coefficients. This means that, for any chosen integer number $k_{0}$, by applying the Theorem 7 , the coefficients

$$
\left\{D H_{j,\left(k_{0}, p\right)}^{(i, 1)}, D H_{j,\left(k_{0}, p\right)}^{(i, 2)}, D H_{j,\left(k_{0}, p\right)}^{(i, 3)} ; 1 \leq i \leq N_{j, p}\right\}
$$

will be reduced to $\left\{D H_{j,\left(k_{0}, p\right)}^{(1,1)}, D H_{j,\left(k_{0}, p\right)}^{(1,2)}, D H_{j,\left(k_{0}, p\right)}^{(1,3)}\right\}$.

From the relations (7), (8), (9), it appears that, each prime number $p$ is characterised by its hybrid wavelet coefficients matrices: $M_{j, p}^{(1)}, M_{j, p}^{(2)}$, $M_{j, p}^{(3)}$, respectively, in $V_{j}^{1} \otimes W_{j}^{1 H}, W_{j}^{1 H} \otimes V_{j}^{1}, W_{j}^{1} \otimes W_{j}^{1 H}$ such as:

$$
\begin{aligned}
& M_{j, p}^{(1)}(i, k)=D H_{j,(k, p)}^{(i, 1)} ; \quad 1 \leq i \leq N_{j, p}, \\
& M_{j, p}^{(2)}(i, k)=D H_{j,(k, p)}^{(i, 2)} ; \quad 1 \leq i \leq N_{j, p},
\end{aligned}
$$




$$
M_{j, p}^{(3)}(i, k)=D H_{j,(k, p)}^{(i, 3)} ; \quad 1 \leq i \leq N_{j, p}
$$

So that, the matrices $M_{j, p}^{(1)}, M_{j, p}^{(2)}, M_{j, p}^{(3)}$ can be written as:

$$
\begin{aligned}
& M_{j, p}^{(1)}=A_{j, p} L_{j, p}^{(1)}, \\
& M_{j, p}^{(2)}=B_{j, p} L_{j, p}^{(2)}, \\
& M_{j, p}^{(3)}=C_{j, p} L_{j, p}^{(3)},
\end{aligned}
$$

where $A_{j, p}, B_{j, p}, C_{j, p}$ are the different orthogonal matrices associated to $W_{j}^{1 H}$, respectively, in $V_{j}^{1} \otimes W_{j}^{1}, W_{j}^{1} \otimes V_{j}^{1}, W_{j}^{1} \otimes W_{j}^{1}$ and $L_{j, p}^{(1)}, L_{j, p}^{(2)}$, $L_{j, p}^{(3)}$ are the classic wavelet coefficients matrices, respectively, in $V_{j}^{1} \otimes W_{j}^{1}, W_{j}^{1} \otimes V_{j}^{1}, W_{j}^{1} \otimes W_{j}^{1}$ such as theirs columns are, respectively,

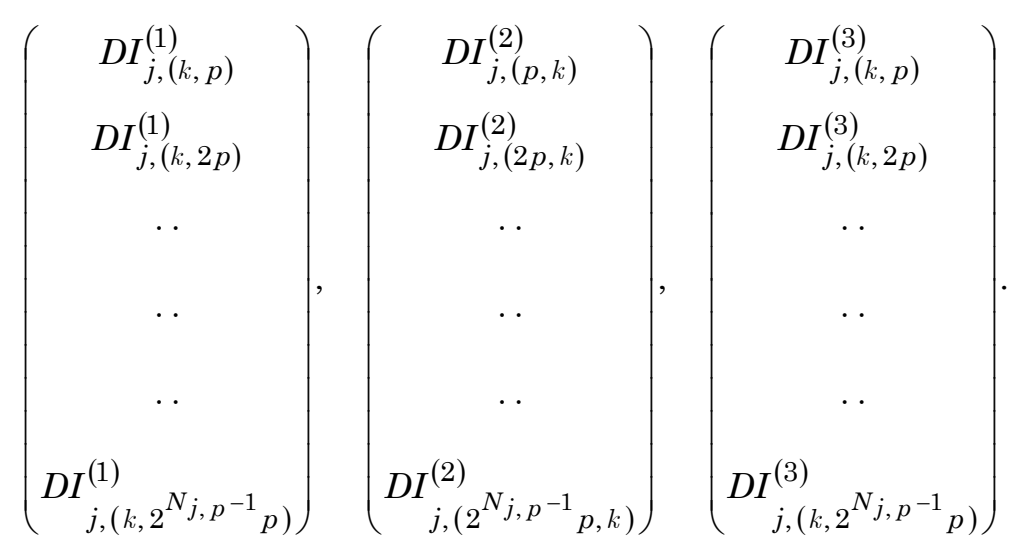

Noting that, the orthogonal matrices $A_{j, p}, B_{j, p}, C_{j, p}$ are constructed in a way of achieving the hybrid wavelet coefficients reduction. This reduction is achieved by applying the Theorem 7 on any single column in $L_{j, p}^{(1)}, L_{j, p}^{(2)}$, and $L_{j, p}^{(3)}$. In addition to this note, the wavelet coefficients of $V_{j}^{1} \otimes W_{j}^{1 H \perp}, W_{j}^{1 H \perp} \otimes V_{j}^{1}, W_{j}^{1} \otimes W_{j}^{1 H \perp}$ are, respectively, arranged in the matrices: $D I_{j}^{(1)} \backslash L_{j, p}^{(1)}, D I_{j}^{(2)} \backslash L_{j, p}^{(2)}, D I_{j}^{(3)} \backslash L_{j, p}^{(3)}$. 


\subsection{The algorithm steps and the corresponding flowchart}

This subsection gives the detailed steps of the (HDS) algorithm with the corresponding flowchart.

Step 1. Loading the image.

Step 2. Decomposition the image with (DWT) at level $j$ produces the matrices: $D I_{j}^{(1)}, D I_{j}^{(2)}, D I_{j}^{(3)}$.

Step 3. This step is composed of five substeps as follows:

- Introducing the prime numbers set and the associated orthogonal matrices ranks $N_{j, p}$.

- From the Step 2, we extract the matrices: $L_{j, p}^{(1)}, L_{j, p}^{(2)}, L_{j, p}^{(3)}$, for each prime number $p$.

- For each prime number $p$, we find the associated orthogonal matrices: $A_{j, p}, B_{j, p}, C_{j, p}$ by applying the Theorem 7 on the first column that has the greatest $\ell^{1}$-norm compared to others columns, respectively, of $L_{j, p}^{(1)}, L_{j, p}^{(2)}, L_{j, p}^{(3)}$.

- For each prime number $p$, we compose the matrices: $M_{j, p}^{(1)}, M_{j, p}^{(2)}$, $M_{j, p}^{(3)}$.

- We arrange all wavelet coefficients, which are contained in $M_{j, p}^{(1)}$, $M_{j, p}^{(2)}, M_{j, p}^{(3)}, D I_{j}^{(1)} \backslash L_{j, p}^{(1)}, D I_{j}^{(2)} \backslash L_{j, p}^{(2)}, D I_{j}^{(3)} \backslash L_{j, p}^{(3)}$.

Step 4. The hard threshold $T(\epsilon, x)$ is applied to all wavelet coefficients such as the coefficients which theirs absolute values are less than $\epsilon$ will be equal to zero, otherwise there is no change.

Step 5. Reconstructing the image through new wavelet coefficients, that are resulted from the thresholding. 
Step 6. The entropy encoder uses a technique to determine the probabilities for each reconstructed image value, so it produces a code based on these probabilities as Huffman encoder, such as more common symbols are generally represented using fewer bits than less common symbols.

Step 7. In this step, we get the compressed image for transmission or storage.

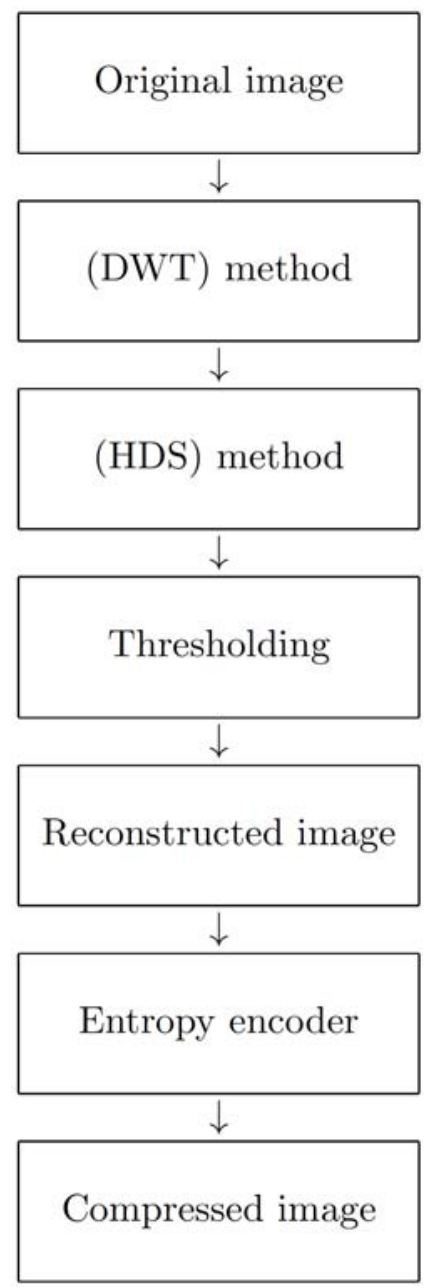

Figure 2. The flowchart corresponding to the (HDS) algorithm. 


\section{Experimental Results and Discussion Using the Hybrid Haar Wavelet}

In order to show the ability of the hybridization of detail space method (HDS) compared to (DWT), it must experiment with a gray-scale image has considerable singularities using the simplest wavelet (Haar wavelet). For that, we have taken an image ('barbara.png') of $240 \times 240$ pixels, its size is $73.8 \mathrm{~KB}$. Considering that the decomposition has been done at level $j=1$, and the algorithm has been implemented in MATLAB with increasing threshold values using the set of all prime numbers which are less than 240 , such as:

$$
2^{N_{0, p^{-1}}} p \leq 240,
$$

where $N_{0, p}$ is the rank of the orthogonal matrix $A_{0, p}$ that characterises the prime number $p$. This rank has been chosen to be the greatest integer number verifies the inequality (10).

So, the compression results with the (HDS) and (DWT) methods have arranged below, respectively, in Table 1 and Table 2 :

Table 1. Compression results with (HDS) method

\begin{tabular}{|c|c|c|c|}
\hline Threshold & PSNR (dB) & Size of compressed image (KB) & CR \\
\hline$\epsilon=20$ & 30.96 & 8.81 & $8.38: 1$ \\
\hline$\epsilon=30$ & 35.66 & 8.35 & $8.84: 1$ \\
\hline$\epsilon=40$ & 40.39 & 8.19 & $9.01: 1$ \\
\hline$\epsilon=50$ & 49.09 & 8.13 & $9.08: 1$ \\
\hline$\epsilon=60$ & 56.88 & 8.12 & $9.09: 1$ \\
\hline
\end{tabular}


Table 2. Compression results with (DWT) method

\begin{tabular}{|c|c|c|c|}
\hline Threshold & PSNR (dB) & Size of compressed image (KB) & CR \\
\hline$\epsilon=20$ & 27.61 & 9.09 & $8.12: 1$ \\
\hline$\epsilon=30$ & 31.20 & 8.55 & $8.63: 1$ \\
\hline$\epsilon=40$ & 35.94 & 8.25 & $8.95: 1$ \\
\hline$\epsilon=50$ & 42.70 & 8.15 & $9.06: 1$ \\
\hline$\epsilon=60$ & 53.62 & 8.13 & $9.08: 1$ \\
\hline
\end{tabular}

These tables present two criteria of images compression. First, the peak signal to noise ratio (PSNR), used to evaluate the quality of the reconstructed image such as, the higher (PSNR) generally produces better reconstructed image quality with the less mean squared error (MSE). Second, the compression ratio (CR), that is used to measure the ability of data compression and to compare many methods by comparing the size of the compressed image to the size of the original image, where the greater (CR) means the less size of compressed image, so the better method.

Obviously, the experimental results indicate that, with the increasing of threshold values, the (PSNR) and (CR) for the two methods increase, such as for each threshold value $\epsilon$, the corresponding (PSNR) and (CR) with the (HDS) method are the greater compared to (DWT) method. So, with the increasing of (PSNR), the reconstructed image quality using the (HDS) method becomes better than the other (DWT) method quality, gradually until the greatest threshold value. Hence, at each threshold value $\epsilon$, the concentration of the wavelet coefficients needed to representing the significative informations of the image with (HDS) method, such as theirs absolute values are less than $\epsilon$ is more than the other with (DWT) method. Although the reconstructed image quality for each threshold value using (HDS) method is better than other quality with (DWT) method, the human eye can not perfectly distinguish the difference between the two images, except for the threshold values 
$(\epsilon=20, \epsilon=30)$ due to that there are grand wavelet coefficients for the (DWT) method more than 30, contain some necessary informations of the original image, where the reconstructed images corresponding to $\epsilon=30$ with the two methods are represented in Figure 5, and the corresponding images difference between the original and reconstructed images are represented in Figure 6. These images difference have accumulated white points, which indicate the singularities of the original image, such as that points of the (HDS) method are less dense and less clarity than that of the (DWT) method, due to that the (HDS) method is more resistant to the singularities than the (DWT). In another side, with the increasing of (CR) for the two methods, the size of the compressed image for each threshold value with the (HDS) method is less than the compressed image size with (DWT) method, where this size with the (HDS) method is the best at the threshold value $(\epsilon=60)$, then the corresponding $(\mathrm{CR})$ is equal to 9.09:1. This greatest (CR) value, means that the compressed image with the (HDS) method occupies only $11 \%$ of the storage space of the original image.

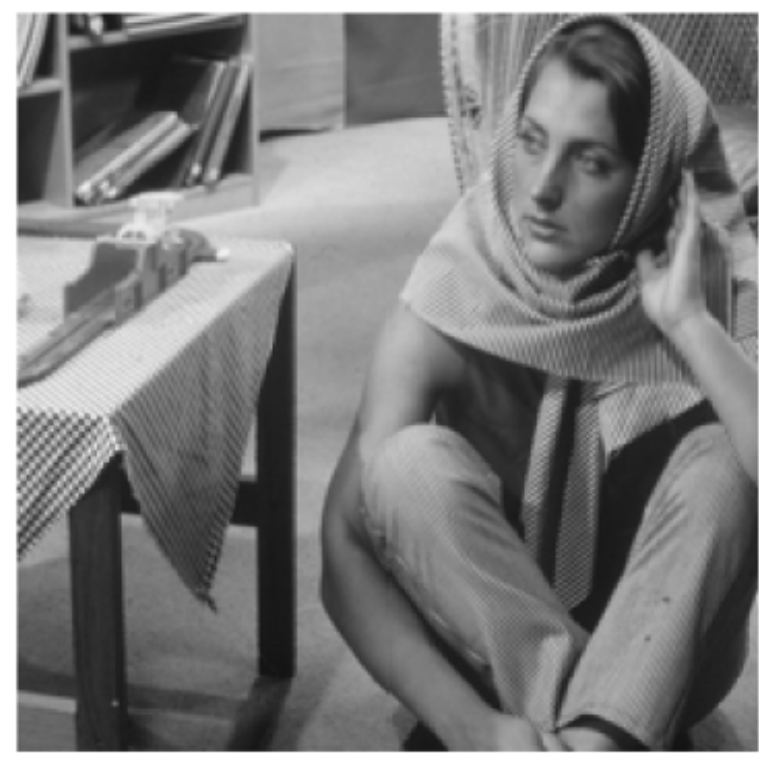

Figure 3. Original image. 

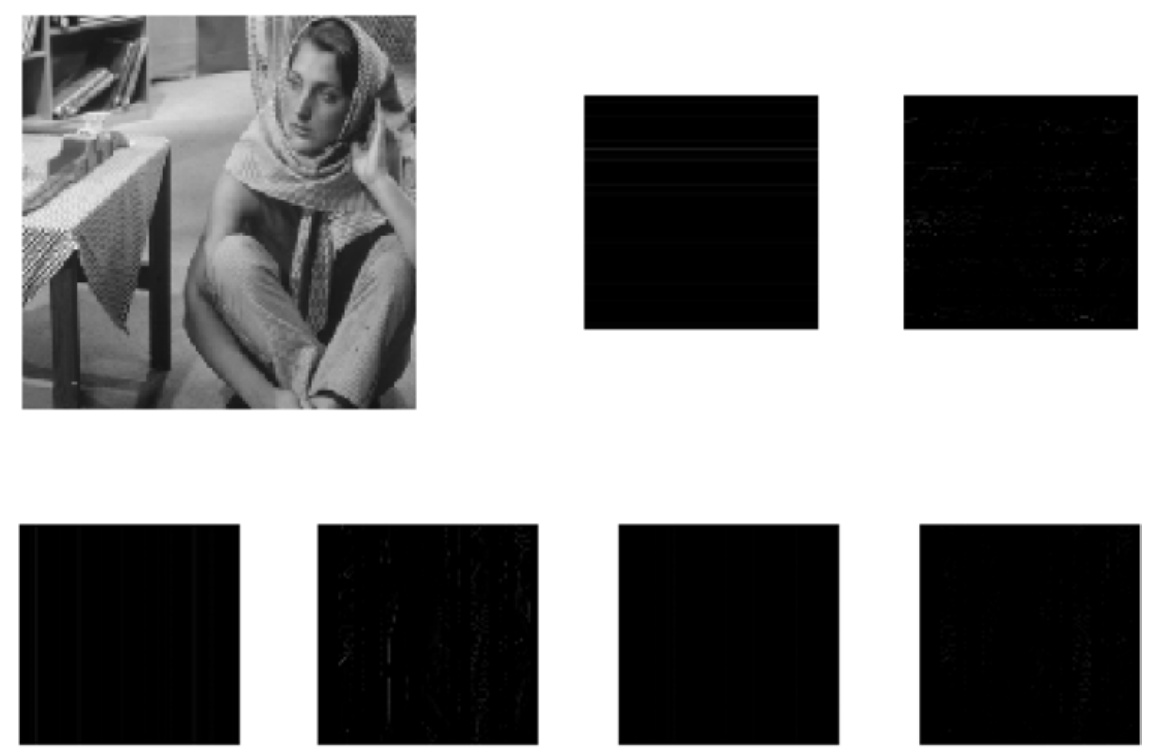

Figure 4. Decomposition image at level $j=1$ before thresholding.

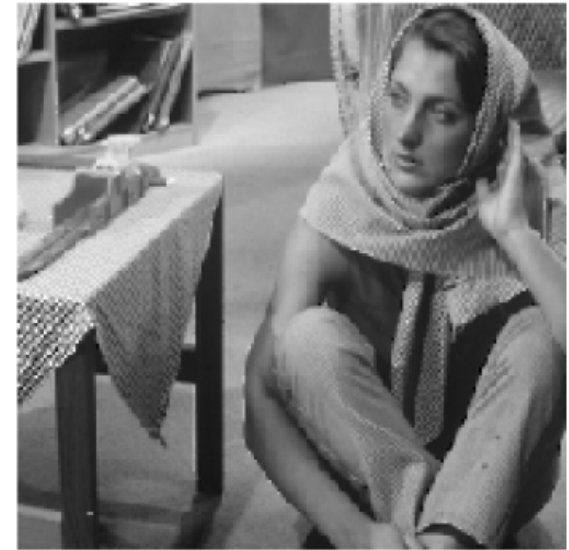

(a)

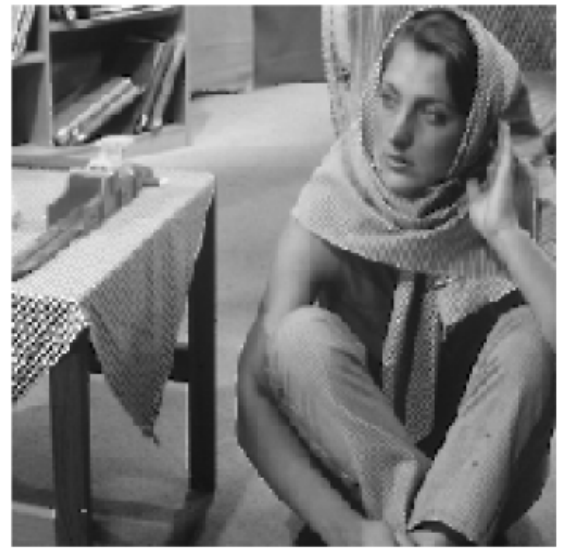

(b)

Figure 5. Reconstructed images, (a) with (HDS) and (b) with (DWT). 


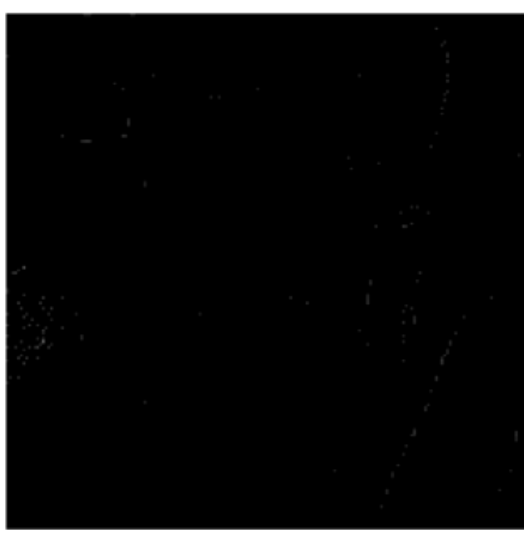

(c)

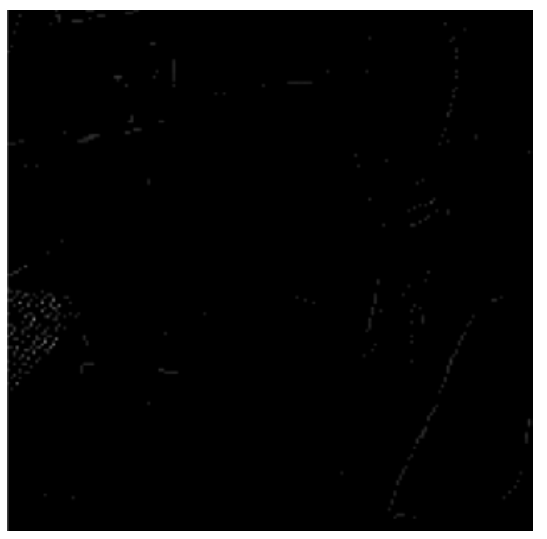

(d)

Figure 6. Images difference, (c) with (HDS) and (d) with (DWT).

\section{Conclusion}

In this paper, a new images compression method based on the hybridization of detail space (HDS) is presented, its advantages compared to (DWT) method have made it adaptive and more responsive to images characterized with large singularities. The experimental results have justified the better performance of this new method such as, high compression ratios with no marked degradation of the compressed image quality. As a result, we can say that the (HDS) method is an efficient images compression and denoising method.

\section{References}

[1] A. Cohen, Numerical Analysis of Wavelet Methods, North-Holland, Studies in Mathematics and its Application 32, 2003.

[2] Cédric Vonesch, Thierry Blu and Michael Unser, Generalized Daubechies Wavelet Families, IEEE Transactions on Signal Processing 55(9) (2007).

[3] Fritz Keinert, Wavelets and Multiwavelets, A CRC Press Company, 2004.

[4] Fuzhen Zhang, Matrix Theory, Springer, New York, Dordrecht, Heidelberg, London, ISBN 9781461410980.

[5] Ingrid Daubechies, Ten Lectures on Wavelets, Society for Industrial and Applied Mathematics, Philadelphia, Pennsylvania, 1992. 
[6] Michel Misiti, Yves Misiti, Georges Oppenheim and Jean-Michel Poggi, Wavelets and their Applications published in France by Hermes Science/Lavoisier in 2003. ISBN-13: 978-1-905209-31-6.

[7] S. Mallat, Multiresolution approximation and wavelet orthonormal bases of L2, Trans. Amer. Math. Soc. 315 (1989), 69-87.

[8] S. Mallat, A Wavelet Tour of Signal Processing, Academic, New York, 1998.

[9] David F. Walnut, An Introduction to Wavelet Analysis, Birkhauser Boston (ISBN0-8176-3962-4) 2002.

[10] Yves Meyer, Wavelets and Operators, Cambridge Univ. Press, Cambridge, U. K., 1992. 